\title{
RESEARCH
}

Open Access

\section{Ontogeny, species identity, and environment dominate microbiome dynamics in wild populations of kissing bugs (Triatominae)}

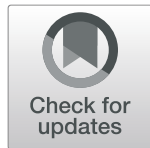

Joel J. Brown ${ }^{1,2 \dagger}$, Sonia M. Rodríguez-Ruano ${ }^{1 \dagger}$, Anbu Poosakkannu' ${ }^{1}$, Giampiero Batani ${ }^{1}$, Justin O. Schmidt ${ }^{3}$, Walter Roachell ${ }^{4}$, Jan Zima Jr ${ }^{1}$, Václav Hypša ${ }^{1}$ and Eva Nováková ${ }^{1,5^{*}}$ (i)

\begin{abstract}
Background: Kissing bugs (Triatominae) are blood-feeding insects best known as the vectors of Trypanosoma cruzi, the causative agent of Chagas' disease. Considering the high epidemiological relevance of these vectors, their biology and bacterial symbiosis remains surprisingly understudied. While previous investigations revealed generally low individual complexity but high among-individual variability of the triatomine microbiomes, any consistent microbiome determinants have not yet been identified across multiple Triatominae species.

Methods: To obtain a more comprehensive view of triatomine microbiomes, we investigated the host-microbiome relationship of five Triatoma species sampled from white-throated woodrat (Neotoma albigula) nests in multiple locations across the USA. We applied optimised 16S rRNA gene metabarcoding with a novel 18S rRNA gene blocking primer to a set of $170 \mathrm{~T}$. cruzi-negative individuals across all six instars.

Results: Triatomine gut microbiome composition is strongly influenced by three principal factors: ontogeny, species identity, and the environment. The microbiomes are characterised by significant loss in bacterial diversity throughout ontogenetic development. First instars possess the highest bacterial diversity while adult microbiomes are routinely dominated by a single taxon. Primarily, the bacterial genus Dietzia dominates late-stage nymphs and adults of T. rubida, T. protracta, and T. lecticularia but is not present in the phylogenetically more distant $T$. gerstaeckeri and T. sanguisuga. Species-specific microbiome composition, particularly pronounced in early instars, is further modulated by locality-specific effects. In addition, pathogenic bacteria of the genus Bartonella, acquired from the vertebrate hosts, are an abundant component of Triatoma microbiomes.
\end{abstract}

(Continued on next page)

\footnotetext{
* Correspondence: novake01@prf.jcu.cz

${ }^{\dagger}$ Joel J. Brown and Sonia M. Rodríguez-Ruano are co-first authors and contributed equally to this MS.

${ }^{1}$ Faculty of Science, University of South Bohemia, Ceske Budejovice, Czech Republic

${ }^{5}$ Institute of Parasitology, Biology Centre of the Czech Academy of Sciences, Ceske Budejovice, Czech Republic

Full list of author information is available at the end of the article
}

(c) The Author(s). 2020 Open Access This article is licensed under a Creative Commons Attribution 4.0 International License, which permits use, sharing, adaptation, distribution and reproduction in any medium or format, as long as you give appropriate credit to the original author(s) and the source, provide a link to the Creative Commons licence, and indicate if changes were made. The images or other third party material in this article are included in the article's Creative Commons licence, unless indicated otherwise in a credit line to the material. If material is not included in the article's Creative Commons licence and your intended use is not permitted by statutory regulation or exceeds the permitted use, you will need to obtain permission directly from the copyright holder. To view a copy of this licence, visit http://creativecommons.org/licenses/by/4.0/ The Creative Commons Public Domain Dedication waiver (http://creativecommons.org/publicdomain/zero/1.0/) applies to the data made available in this article, unless otherwise stated in a credit line to the data. 


\begin{abstract}
(Continued from previous page)
Conclusion: Our study is the first to demonstrate deterministic patterns in microbiome composition among all life stages and multiple Triatoma species. We hypothesise that triatomine microbiome assemblages are produced by species- and life stage-dependent uptake of environmental bacteria and multiple indirect transmission strategies that promote bacterial transfer between individuals. Altogether, our study highlights the complexity of Triatominae symbiosis with bacteria and warrant further investigation to understand microbiome function in these important vectors.
\end{abstract}

Keywords: Bacteria, Blood, Hematophagous, Insect, Microbiome, Ontogeny, Pathogen, Triatominae, Vector

\section{Background}

Kissing bugs (Hemiptera: Reduviidae: Triatominae) are hemimetabolous blood-feeding insects predominantly found across the Americas. They are the vectors of Chagas' disease $(\mathrm{CD}$; caused by the trypanosomatid parasite Trypanosoma cruzi) which the majority of $\sim 150$ known species can transmit to a wide range of mammalian hosts, including humans [1, 2]. There are 11 endemic North American species, whose epidemiological relevance has been overlooked compared to their neotropical relatives. However, multiple recent studies have recorded high prevalence of $T$. cruzi in kissing bugs and reservoir mammals, like packrats, racoons, and opossums [3-5], and others have confirmed cases of autochthonous human CD in the USA [6-10]. Thus, T. cruzi transmission by native US vectors has become a current health concern, emphasising the need for in-depth understanding of triatomine vector biology.

Hematophagous (blood-feeding) organisms are broadly affected by their associated microbial communities (referred to as the "microbiome"). Microbiome diversity, composition, and function directly influence various fundamental aspects of host biology, such as immunity, thermal tolerance, and digestion [11-13]. Nutritionally, blood is rich in proteins and salt, lacks vitamins, and its breakdown releases toxic amounts of haem and urea [14, 15]. Many symbiotic bacteria facilitate blood meal digestion and synthesise essential vitamins, making them important mutualists for their hosts [16-23]. Furthermore, the gut bacteria of hematophagous vectors interact with parasites (like T. cruzi) occupying the same niche [2, 24, 25]. The microbiome can potentially impede parasite transmission through direct (competition for resources) and indirect (promoting immune response) interactions [26-32]. The most comprehensive background on hematophagous microbiomes has been derived from mosquitos, ticks, and tsetse flies (reviewed in [33]), whereas triatomine-bacteria associations remain neglected.

To establish a basic background for studying the dynamics and potential function of Triatominae microbiomes, we need to elucidate the main factors determining their composition. In other systems, microbiomes usually display species specificity, i.e., they differ even among closely related host taxa (e.g., [34]). In some cases, the differences reflect the phylogenetic relationships of the hosts (phylosymbiosis, e.g., [35]). On the other hand, since environment is a natural source of at least some portion of the microbiomes, the habitat and geographic location of the host may significantly affect microbiome composition (e.g., [36-39]). In triatomines, thirteen high throughput sequencing studies published since 2015 have indicated a wide range of factors that potentially influence their microbiomes. However, it remains difficult to derive any consistent cross-species patterns since the studies utilised a wide variety of DNA templates, including pooled or individual bodies, entire abdomens, the distal part of the abdomen, whole guts, midguts, faeces, and cultured bacterial colonies [40-52], and were often further complicated by other variables (e.g., sex, locality, instar, T. cruzi infection status). Apart from these methodological differences, the disparity among studies could also reflect true biological characteristics of Triatominae. These include lengthy development times through five nymphal instars [48-52], complex physiology of the alimentary tract [48], and accessory feeding strategies, like haemolymphagy (feeding on arthropod haemolymph), kleptohematophagy (stealing a blood meal from another triatomine), and conspecific coprophagy (feeding on faeces) known to be employed by some triatomine species [2, 53-56].

To date, only a single study [52] has sampled microbiomes from multiple wild populations of any triatomine species. Others have mostly targeted South American vectors in domestic environments or laboratory-reared specimens [44-49], with little consideration for nonurban systems. In this study, we thus focus on wild populations of 5 Triatoma species in southern Texas and Arizona. Sampling triatomines within the nests of a favoured host, the white-throated woodrat (Neotoma albigula), substantially increases the probability of an identical blood source (a factor known to influence microbiome composition [57-59]) and allows us to collect all five instars and adults. Furthermore, centring this study on T. cruzi-negative individuals eliminates another variable known to influence microbiome composition. 
Our controlled design thus provides the opportunity to (i) evaluate microbiome development across the full ontogenetic spectrum (first to fifth instars plus adults) in natural populations, (ii) determine any relationship between triatomine genetic background and microbiome diversity, (iii) examine an environmental effect on microbiome composition in species from multiple distinct geographic areas, (iv) determine microbiome specificity among Triatoma species from the same microhabitat, and (v) identify pathogens acquired through feeding on the vertebrate host.

\section{Methods}

\section{Study sites and sample set}

Samples were collected from 3 sites in southern Texas in July 2017 (Chaparral Wildlife Management Area, Camp Bullis and Lackland Air Force base in San Antonio) and 3 sites in southern Arizona in July 2018 (Las Cienegas National Conservation Area, University of Arizona Desert Station in Tucson, and San Pedro Riparian National Conservation Area). Sites were accessed with full written permission from the relevant governing bodies (see the "Acknowledgements" section). Larval instars and adults, molecularly determined as Triatoma rubida $(N=128)$, $T$. lecticularia $(N=20), T$. sanguisuga $(N=25), T$. gerstaeckeri $(N=42)$, and $T$. protracta $(N=34$; see below), were collected from the nests of white-throated woodrats (Neotoma albigula). We recorded nest coordinates, developmental stage, morphospecies, engorgement, and sex (adults only) for every individual. All samples were preserved in individual vials with $100 \%$ ethanol. Additional samples of adult $T$. protracta and $T$. rubida were provided from two houses neighbouring the University of Arizona Desert Station. Since these were adult individuals attracted by black light and not a permanent domestic infestation, we included them in the study.

\section{DNA extraction and basic molecular analyses}

The entire abdomen (comprising the whole gut length) of each individual sample was used as a template for DNA isolation with DNeasy Blood and Tissue kits (Qiagen) according to manufacturer instructions. DNA templates were stored at $-75^{\circ} \mathrm{C}$ prior to molecular analyses, which included host molecular taxonomy, T. cruzi infection status, and $16 \mathrm{~S}$ rRNA gene amplicon library preparation. To determine Triatoma species identity and phylogenetic distance we used the primers 7432F (5'-GGACGWGG WATTTATTATGGATC-3') and 7433R (5'-GCWC CAATTCARGTTARTAA-3') to amplify a $663 \mathrm{bp}$ fragment of the $c y t B$ gene [60]. However, the primer pair $7432 \mathrm{~F}$ and $7433 \mathrm{R}$ failed to amplify a PCR product in samples morphologically identified as $T$. protracta, for which some difficulties with $c y t B$ sequencing have previously been reported [61, 62]. We therefore designed alternative primers, Tpr_F (5'CCTACTATCCGCGGTTCCTT-3') and Tpr_R (5'GGGATGGATCGGAGAATTGCG-3') using three available $T$. protracta $c y t B$ sequences and seven sequences of different Triatoma species from GenBank. Under the same conditions published by Monteiro et al. [60] the amplification resulted in 380-bp long sequences. The PCR products were cleaned from primers using Exonuclease I and FastAP (Thermo Scientific) enzymes and Sanger sequenced. Phylogenetic background of the Triatoma spp. sample set was reconstructed from aligned sequences using maximum likelihood with the best fitting model determined by a corrected Akaike information criterion in jModelTest2 [63]. Representative sequences for each species are available in GenBank under the following accession numbers MT239320-MT239329.

To eliminate infection status as a variable affecting the host microbiome, all samples were screened for the presence of T. cruzi in three PCR reactions (as described in Rodríguez-Ruano et al. [51]). In brief, T. cruzi presence/ absence was confirmed with the universal primer pair TCZ1/TCZ2, targeting any discrete typing unit (DTU) as described by Moser et al. [64]. Additionally, two primer sets (ME/TC1 or TC2) were used to distinguish different discrete typing units of $T$. cruzi $[40,65]$. The representative PCR products of all three primer pairs were Sanger sequenced (as described above) and their identity evaluated based on BLASTn searches to confirm the specificity of the screening process. All bands of the expected size from the PCR products were identified as T. cruzi DTUs. 57 T. cruzi-positive samples were subsequently excluded from the analyses. The complete metadata for the samples used in this study are provided in Additional File 1.

\section{Amplicon library preparation}

Extracted DNA templates were used for 16S rRNA gene amplification according to Earth Microbiome Project standards (EMP; http://www.earthmicrobiome.org/protocols-and-standards/16s/). Sample multiplexing was based on a double barcoding strategy with EMPproposed 12-bp Golay barcodes included in the forward primer 515F [66], and additional 5-bp barcodes (designed in our laboratory) within the reverse primer 926R $[66,67]$. Barcodes and primers are available in Additional File 1. The resultant amplicons were approximately $500 \mathrm{bp}$ long, including adapters, barcodes, primer sequences, and approximately $400 \mathrm{bp}$ of the 16S rRNA gene V4/V5 hypervariable region. 


\section{Novel 18S rRNA gene blocking primer}

Since our previous sequencing of insect-associated microbiomes with the 515F/926R primer pair $[66,67]$ revealed low specificity towards the $16 \mathrm{~S}$ rRNA gene (resulting in high numbers of host 18S rRNA gene amplicons; unpublished data) we implemented a custom $18 \mathrm{~S}$ rRNA gene blocking primer (see [68] for pertinent application of a blocking primer). The $8 \mathrm{bp}$ at the $5^{\prime}$ end of the blocking primer 926X (5' GTGCCCTTCCGTCA ATTCCT-C3 3') were designed to specifically match the $18 \mathrm{~S}$ rRNA gene sequence (conserved in representatives of 23 Insecta orders, a human, and a mouse; Additional File 1), while the last 12 bp partially overlaps with the 926R (5' CCGYCAATTYMTTTRAGTTT 3') annealing site. The $\mathrm{C} 3$ spacer modification at the $3^{\prime}$ end of 926X was introduced to prevent any prolongation of the blocker. In addition, the blocking primer was used at $10 \times$ higher concentration compared to that of the amplification primers $[68,69]$. This concentration disparity results in the $926 \mathrm{R}$ primers being outcompeted by the blocker 926X during any possible annealing to the $18 \mathrm{~S}$ rRNA gene, thus increasing the $16 \mathrm{~S}$ rRNA gene amplification efficiency (detailed information on blocking primer design and validation are provided in Additional File 2).

\section{Library controls and amplicon sequencing}

In order to confirm the barcoding output and evaluate any effect of our blocking primer on 16S rRNA gene amplification (e.g., possible amplification bias towards some bacterial taxa), the library contained two types of commercially available microbiome mock communities and three microbiome samples of colony-reared Rhodnius prolixus sequenced in our previous projects [51]. The mock communities comprised three samples of gDNA templates with an equal composition of 10 bacterial species (ATCC MSA-1000 ${ }^{\circ \mathrm{m}}$ ) and three samples with staggered composition of the same 10 bacteria $\left(\right.$ ATCC $^{\bullet}$ MSA- $\left.^{-1001^{\mathrm{mx}}}\right)$. Altogether seven negative controls $(\mathrm{NC})$ were used to control for the extraction procedure $(2 \mathrm{NC})$, PCR library preparation $(2 \mathrm{NC})$, and well-to-well contamination (3 NC: PCR water template). The PCR amplicons were cleaned using AMPure XP (Beckman Coulter) magnetic beads and equimolarly pooled based on DNA concentration measured with a Synergy H1 (BioTek) spectrophotometer. Since the bead purification did not completely remove the high concentrations of the blocking primer, the final pooled library was purified using Pippin Prep (Sage science) in order to remove all DNA fragments shorter than $300 \mathrm{bp}$ and longer than $1100 \mathrm{bp}$. The purified library was sequenced in a single run of Illumina MiSeq using v3 chemistry with $2 \times 300$ bp output (Norwegian High Throughput Sequencing Centre, Department of Medical Genetics, Oslo University Hospital).

\section{Sequence processing}

The raw reads were quality checked (FastQC) and trimmed (necessary for reverse reads due to the reduced end-of-read quality) using USEARCH v9.2.64 [70]. The reads were processed according to the following workflow, implementing suitable scripts from USEARCH v9.2.64 [70]. Pair-end reads were demultiplexed and merged. Primers were trimmed and sequences were quality filtered, resulting in a final amplicon length of $369 \mathrm{bp}$. The dataset was clustered at $100 \%$ identity to get a representative set of sequences for de novo OTU picking, using the USEARCH global alignment option at $97 \%$ identity match [70]. Taxonomy was assigned to the representative sequences using the BLAST algorithm [71] against the SILVA 132 database trimmed for the SSU rRNA gene [72]. Chloroplast sequences, mitochondrial OTUs, and singletons were removed from the final OTU table using QIIME 1.9 [73].

We analysed the final OTU table as three independent datasets, to make sure that our bioinformatic approach did not influence the results. We made the "basic" dataset (567 OTUs) by filtering extremely low abundant OTUs (as recommended by Bokulich et al. [74]). We generated the "decontam" dataset (5553 OTUs) from the final OTU table by filtering potential contaminants, using the R package "decontam" (V1.5.0) [75] to systematically identify and discard a total of 118 OTUs (complete list in Additional File 1) with a frequencybased approach combined with the post-PCR concentration of each sample. Three of the computationally identified contaminant OTUs (one assigned to the genus Sphingomonas and two to Geobacillus) were present in all of our negative controls, comprising $223 \pm 195$ mean total bacterial reads. We generated the "ultraclean" dataset (183 OTUs) from the "decontam" dataset by employing stringent filtering steps to reduce data complexity, based on our previous experience with insect microbiomes. We retained the OTUs that met the following conditions: first, representing more than $1 \%$ of reads in any individual sample, and second, being found repeatedly, i.e. in at least two samples across the dataset.

\section{Statistical analyses}

We carried out all downstream analyses on the three normalised datasets using rarefaction at 1000 sequences per sample for "basic" and "decontam", and 500 sequences per sample for "ultraclean". We used the "vegan" [76] and "phyloseq" [77] packages in R [78] to calculate community quantitative measures (Richness and Shannon index) and ordination analyses (non-metric multidimensional scaling, NMDS; based on Bray-Curtis dissimilarities). We supported the ordination analyses using PERMANOVA tests with beta-dispersion to 
determine the significance of the tested factors on shaping the microbiome composition.

To test the effect of ontogeny, we analysed the microbiome communities across host developmental stages in T. rubida, T. protracta, T. lecticularia, and T. gerstaeckeri. To test for differentiation in microbiome communities with a distinct geographic background, we analysed T. rubida samples from two different locations in Arizona. We assessed possible species-specific differentiation by comparing individuals from all 5 species, and by comparing T. gerstaeckeri and T. lecticularia collected from the same nest in southern Texas (thus eliminating the geographic variable). We evaluated the possible effect of host phylogeny on species-specific microbiome patterns. Using Mantel test (implemented in the R package "ecodist" [79]), we tested correlations between microbiome Bray-Curtis dissimilarities and host genetic distance (obtained using neighbour-joining analysis with Tamura-Nei model for $c y t B$ alignment with discarded gap and ambiguous positions). The same approach was used to evaluate the effect of geographic distance among sampling sites calculated in QGIS [80].

\section{Results}

\section{Molecular data}

The Illumina MiSeq run generated 11,991,455 reads. With negative controls removed, we retrieved a mean average of 13,883 reads per sample and a median average of 9398 reads. In our positive controls, we retrieved consistent profiles of expected diversity from the commercially produced mock communities (Additional File 1). Two of the staggered mocks lacked one taxon with $0.04 \%$ abundance. The composition of equal mocks was consistently biassed towards an overrepresentation of Clostridium (2.8 times the expected value of $10 \%$ ), which led to $0.5-8.6 \%$ decreases in other taxa. Within the staggered communities, we retrieved most taxa in the expected proportions (from $0.04 \%$ to $44.78 \%$ ). Three of the low abundant taxa (Clostridium, Lactobacillus, and Streptococcus) were overrepresented. The most underrepresented component was Rhodobacter (see Additional File 1). All three Rhodnius prolixus positive controls showed consistent profiles: Enterococcus (mean $(\mathrm{SD})=$ 86(2)\%), Bacillus (mean(SD) = 10(1)\%), and Arsenophonus $($ mean $(\mathrm{SD})=4(1) \%)$, which matched the results of our previous sequencing runs [51].

The results focus on $170 \mathrm{~T}$. cruzi-negative samples from the "ultraclean" dataset (see section 2.6; metadata available in Additional File 1). The corresponding results of ordination analyses from the "basic" and "decontam" datasets are available in Additional File 3. Phylogenetic clustering based on maximum likelihood (Additional File 4) unequivocally determined the 170 samples from "ultraclean" to be $T$. rubida $(N=81)$, T. lecticularia $(N$ $=13), T$. sanguisuga $(N=15), T$. gerstaeckeri $(N=27)$, and $T$. protracta $(N=34)$.

\section{Microbiome dynamics and host ontogeny}

Host ontogeny is a major factor influencing triatomine microbiomes (Fig. 1a-c). T. rubida (the most abundant species in our data) shows a pronounced pattern of diversity loss and Dietzia accumulation throughout ontogenetic development. Dietzia is absent in our "ultraclean" data from the earliest instars, progresses into some L3s, and then clearly increases in L4 nymphs. In most adults it completely dominates the microbiome, comprising $100 \%$ of the reads in some individuals (Fig. 1a). The same ontogenetic pattern exists in T. gerstaeckeri, T. protracta, and T. lecticularia but was not analysed with statistical support due to smaller sample sizes and some instar unavailability (see Additional File 5). Furthermore, there were significant differences in T. rubida microbiome diversity between early and late life stages (6 pairwise comparisons retrieved significant differences at the 0.001 confidence interval, Fig. 1b). First instars had the highest Shannon index value (L1 median average $=2.75$ ) and adults had the lowest (L6 median average = $0.01)$. In a non-metric multidimensional scaling analysis (NMDS), T. rubida microbiomes clustered into significantly distinct groups reflecting their ontogenetic development (Fig. 1c; mean stress $\approx 0.16$; PERMANOVA $R^{2}=$ $0.288, p \leq 0.001$, with significant beta-dispersion $\mathrm{F}=$ 3.252, $\mathrm{p}=0.014$ on 999 permutations). A single $T$. rubida L3 outlier with $100 \%$ reads from Streptobacillus (Additional File 1) has been removed from our analyses.

The results presented in Fig. 2 are for two ontogenetic subsets: early (L1-L3) and late (L4-L6) stages, based on their significantly different variance (Additional File 6). There was high among-individual variation in microbiome diversity for all species and instars (Fig. 1a and Additional File 5) but all harboured bacteria from two classes, Actinobacteria and Gammaproteobacteria (Fig. 2). We found species-specific patterns in microbiome composition: only $T$. sanguisuga and $T$. gerstaeckeri contained Acidobacteria and TM6 class bacteria, and only T. protracta possessed Bacteroidia in high abundance (particularly the genus Proteiniphilum, which dominated some adults; Additional File 5). At the genus level, Dietzia dominated the later developmental stages of T. protracta, T. lecticularia, and T. rubida, yet was completely absent from T. sanguisuga and T. gerstaeckeri (Fig. 2). Specifically, Dietzia comprised 2 OTUs (62\% prevalence; abundance median $[\min -\max ]=31.6 \%[0-100 \%])$ in $T$. protracta, 2 OTUs (77\% prevalence; abundance median $[\min -\max ]=79.0 \%[0-100 \%])$ in T. lecticularia, and 3 OTUs $(29 \%$ prevalence; abundance median $[\min -\max ]=$ $0.0 \%$ [0-100\%]) in T. rubida. In contrast, T. sanguisuga 
A
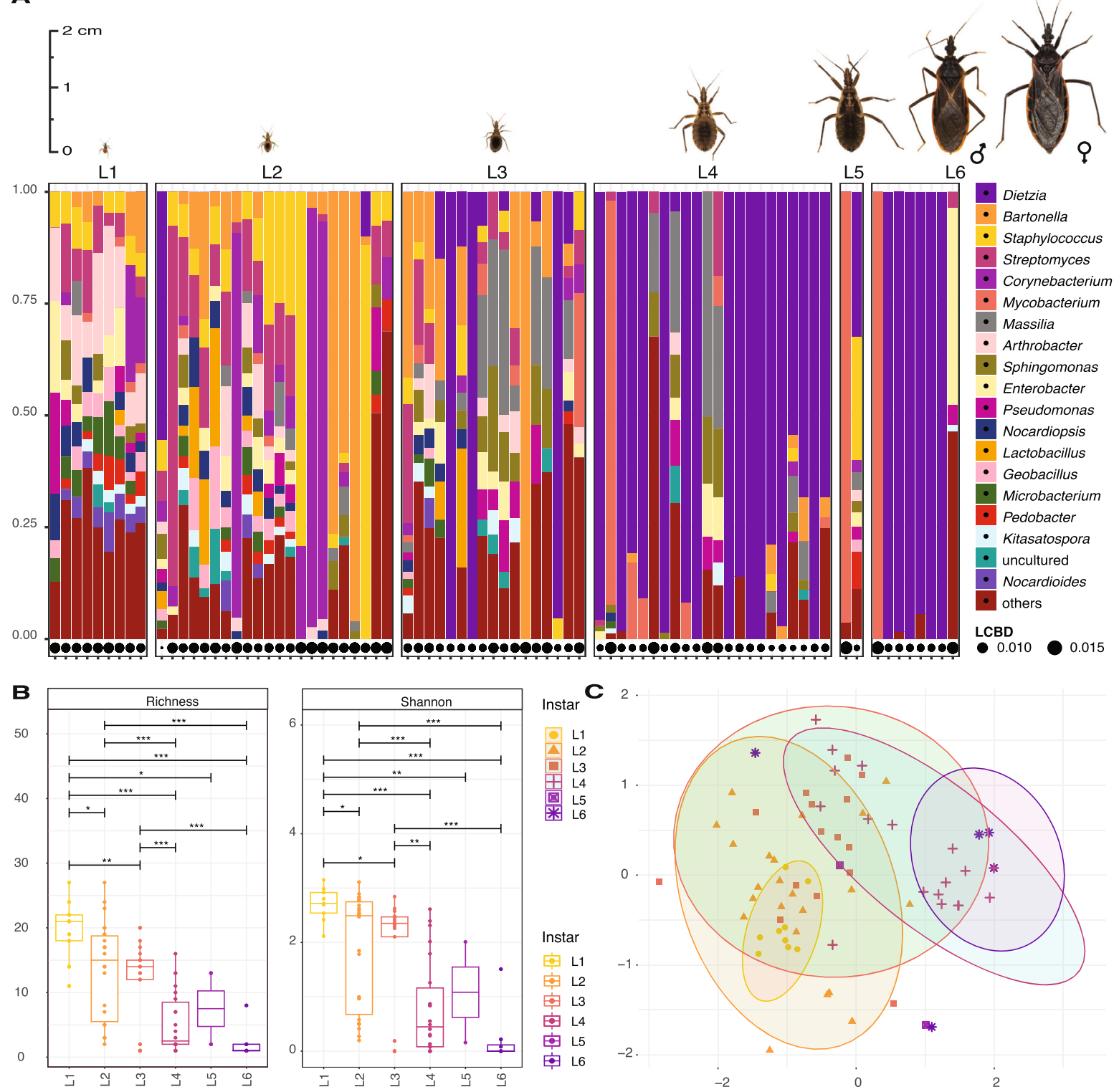

Fig. 1 Ontogenetic shift of Triatoma rubida microbiomes in sylvatic populations from Arizona. Pictures of each T. rubida life stage are to proportional scale. a The microbiome profiles showing the 20 most abundant bacterial genera, listed in decreasing order of abundance to the right of the figure. Each column represents a single individual. LCBD, local contributions to beta diversity. The size of the circle for each sample indicates the degree of uniqueness for that sample relative to the overall variation in community composition [81]. b Richness and Shannon index ( $Y$ axis) for each developmental stage $(X$ axis). Each point represents an individual bug. Length of the horizontal bar indicates the two stages being compared. Asterisks designate significance level as follows $*^{* *} \leq 0.001,{ }^{* *} \leq 0.01,{ }^{*} \leq 0.05$. c NMDS on Bray-Curtis dissimilarity values for microbiomes of different developmental stage. Each colour and shape correspond to a different instar; L6 stands for adults. Each point represents an individual bug. Ellipses are statistically significant at 0.05 confidence interval

was dominated by the genus Streptomyces (5 OTUs; 75\% prevalence; abundance median $[\min -\max ]=19.1 \%[0-$ 90\%]), as was T. gerstaeckeri (6 OTUs; 93\% prevalence; abundance median $[\min -\max ]=23.6 \%[0-90 \%])$.

\section{Microbiome and host genetic background}

We found species-specific differences between the microbiome communities of all five Triatoma species
(Fig. 3). Evaluation of early instar microbiomes confirmed significant differences among clusters reflecting host species identity (NMDS ordination, mean stress $\approx 0.02$; PERMANOVA, $R^{2}=0.18, p \leq 0.001$, betadispersion on 999 permutations: $p=0.034$, Fig. 3). To further test host species specificity in microbiome composition, we specifically compared $T$. gerstaeckeri (17 individuals) and $T$. lecticularia (13 individuals) 


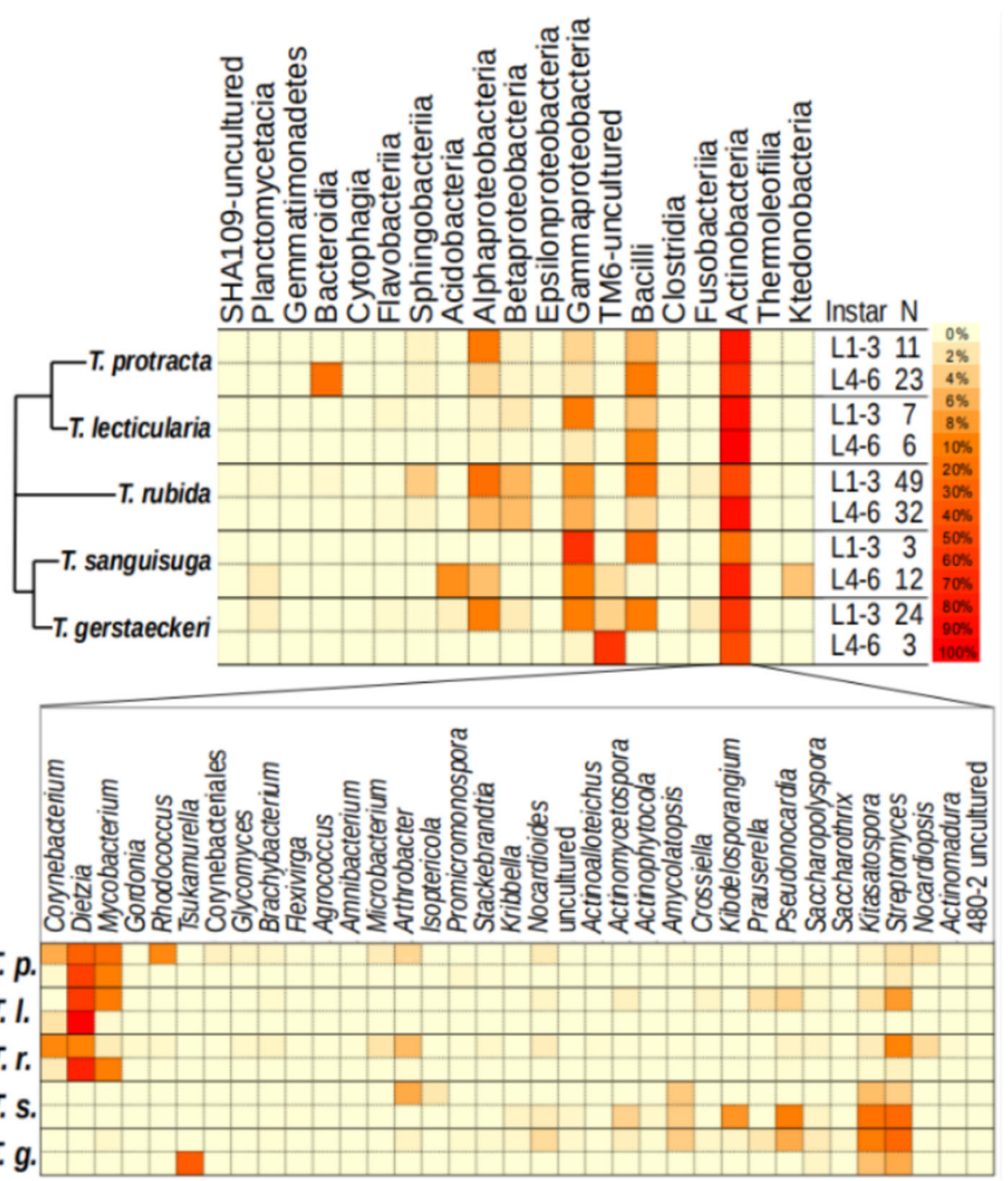

Fig. 2 Microbiome profiles of five Triatoma species. The top heatmap representing the microbiome profile at order level for early and late instar ranges of all Triatoma species. The percentage of each taxon was calculated and normalised by the sum relative abundance per sample, divided by sample size $(N)$. The simplified phylogenetic scheme for the five Triatoma species is based on Georgieva et al. [5] and Ibarra-Cerdeña et al. [82], indicating an uncertain position of T. rubida. The bottom heatmap represents the genus-level diversity found within the most abundant order (Actinobacteria) present in all species

sampled simultaneously from the same $N$. albigula nest in Chaparral, Texas. These species formed distinct clusters with all individuals included in the analysis (NMDS with mean stress $\approx 0.09$; PERMANOVA $R^{2}=0.268, p=0.002$, beta-dispersion on 999 permutations: $p=0.022$; Fig. 4 ). When we analysed the 23 early instar (L1-L3) individuals, we found the same distinct clusters (NMDS with mean stress $\approx$ 0.11; PERMANOVA $R^{2}=0.150, p \leq 0.001$, betadispersion on 999 permutations: $p=0.522$ ). They notably differed in microbiome taxonomic composition, with Dietzia conspicuously absent from $T$. gerstaeckeri but highly abundant in T. lecticularia (Fig. 4).
In addition, we performed two-sided Mantel tests on 93 early instars (L1-L3) to determine if species-specific microbiome differences were a product of host phylogenetic constraint. We identified positive correlations between microbiome dissimilarities and respective host phylogenetic distances (Spearman's rank correlation: $r$ $=0.29, p \leq 0.001$ ). We illustrated this result with a neighbour-joining tree of Bray-Curtis microbiome dissimilarities that specifically included triatomine species identity and geographic origin (Fig. 5). T. sanguisuga and $T$. gerstaeckeri microbiomes were arranged in a single cluster which reflects the host's close phylogenetic relationship. Microbiomes of T. lecticularia predominantly clustered according to host phylogeny 


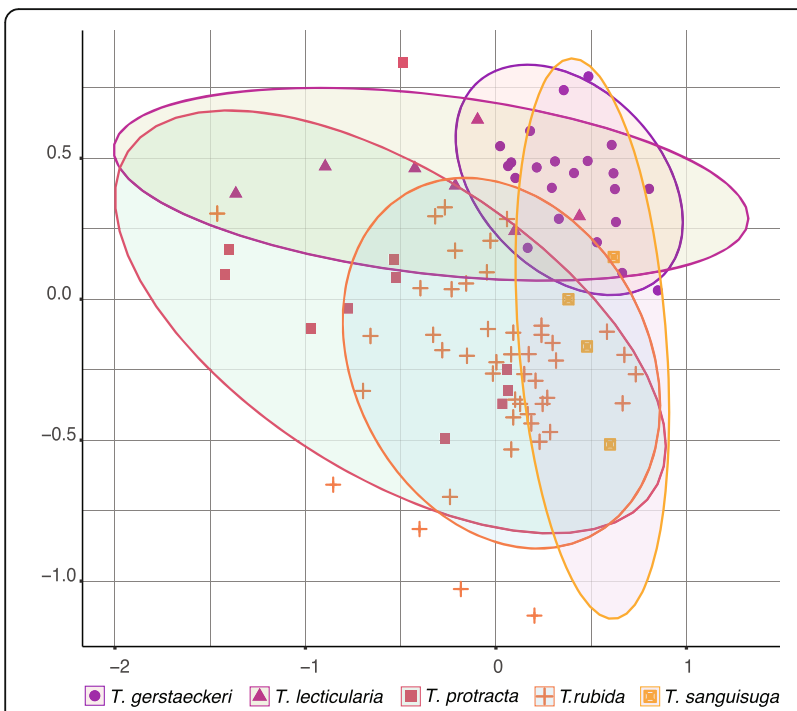

Fig. 3 Triatoma microbiome species specificity. NMDS ordination for early instars (L1-L3) of all five Triatoma species (T. rubida, $T$. protracta, T. gerstaeckeri, T. sanguisuga, and T. lecticularia) based on Bray-Curtis dissimilarities of their microbiome communities. Each point is an individual bug. Each species is represented by a distinct colour and shape shown beneath the $x$ axis

(close to $T$. protracta samples), despite the common geographic origin of $T$. sanguisuga, $T$. gerstaeckeri, and T. lecticularia. T. protracta branching was affected by both phylogeny and geographic origin. Overall, both phylogeny and geographic origin partially explain microbiome composition. Analysis of late instars (74

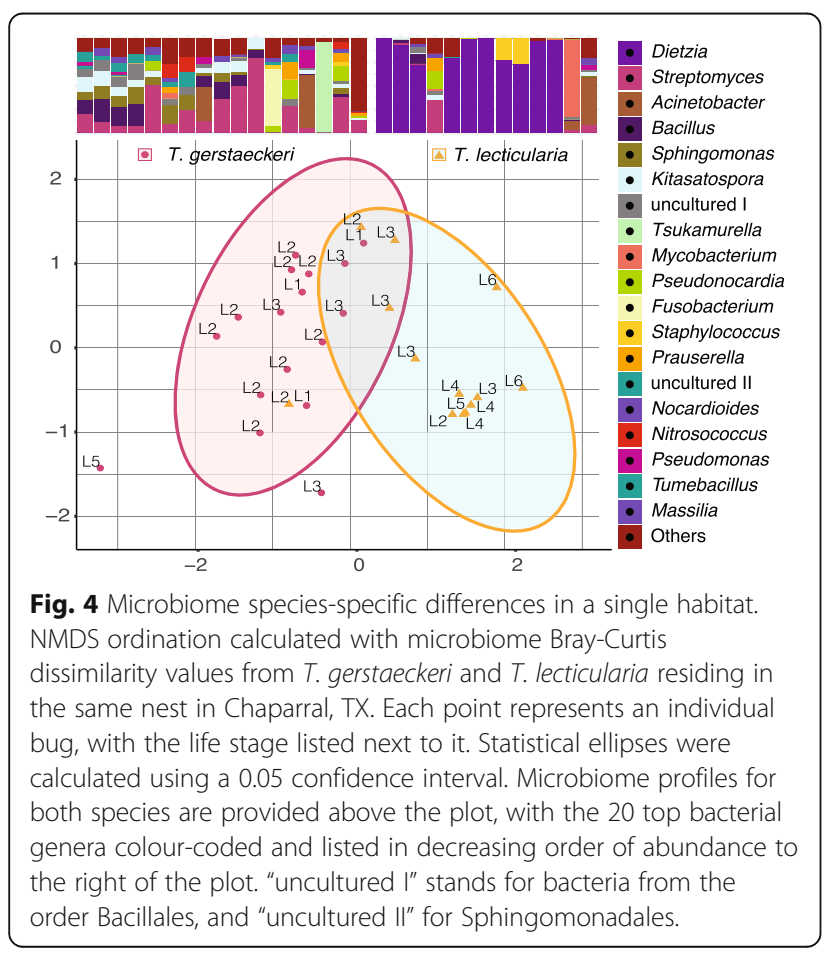

individuals L4-L6) resulted in a notably lower degree of correlation (Spearman's rank correlation: $r=0.18$, $p \leq 0.001)$.

\section{Microbiome and host geographic origin}

Geographic location was a small but significant factor explaining microbiome variation at the intra-species level. We demonstrated this by comparing $T$. rubida from nests in two Arizona locations (Las Cienegas National Conservation Area (LCNCA) and University of Arizona Desert Station (UADS)). We grouped them into early and late instar ranges, to account for ontogenetic changes in microbiome composition, and found their microbiomes significantly differed based on locality. NMDS (mean stress $\approx 0.17$ ) showed statistically significant clusters (PERMANOVA, $R^{2}=0.08, p \leq 0.001$, with non-significant beta-dispersion on 999 permutations, $F$ $=0.393, p=0.537$; Fig 6).

Furthermore, we analysed the microbiomes of $21 \mathrm{~T}$. rubida from 6 different nests within UADS to see if $N$. albigula nests function as natural isolated microhabitats, potentially structuring the population variability among T. rubida microbiomes. Our results show that microbiome variability reflected the nest origin among early instars (L1-L3), supported with statistically significant clusters in the NMDS analysis (PERMANOVA, $R^{2}=$ $0.45, p \leq 0.001$; Additional File 7) and a modest correlation with geographic distance between the nests (Mantel test, Spearman's rank correlation: $r=0.164, p=$ 0.019 , at $95 \%$ confidence).

\section{Inherited taxa and bacterial pathogens}

Sampling across the ontogenetic spectrum allowed us to examine bacterial taxa shared between adult triatomines and their presumed progeny. For this analysis, we only considered adults and early instar individuals originating from the same nest and sharing 100\% identity among their $\operatorname{cox} B$ gene sequences. Cross-referencing our "decontam" unrarefied data (to deliberately include low abundance taxa that were excluded in the "ultraclean" dataset) with early and late developmental stages of $T$. rubida (comparison between three L2s and one adult from nest number 4 in LCNCA, AZ) indicated 17 shared OTUs with abundance $>0.05 \%$ per sample, associated with all individuals. 11 OTUs from Actinobacteria represented the genera Dietzia, Mycobacterium, Corynebacterium, Brachybacterium, Amycolatopsis, Kitasatospora, Nocardiopsis, and Streptomyces (4 OTUs); 1 OTU of an uncultured bacterium from Bacteriodetes; and 5 OTUs from Firmicutes (Geobacillus, Staphylococcus, Lactobacillus (2 OTUs), and Ruminococcus). For T. lecticularia (comparison between three L2 individuals and two adults from nest number 2 in Chaparral, TX), we found 4 shared OTUs with abundance $>0.05 \%$ per sample 


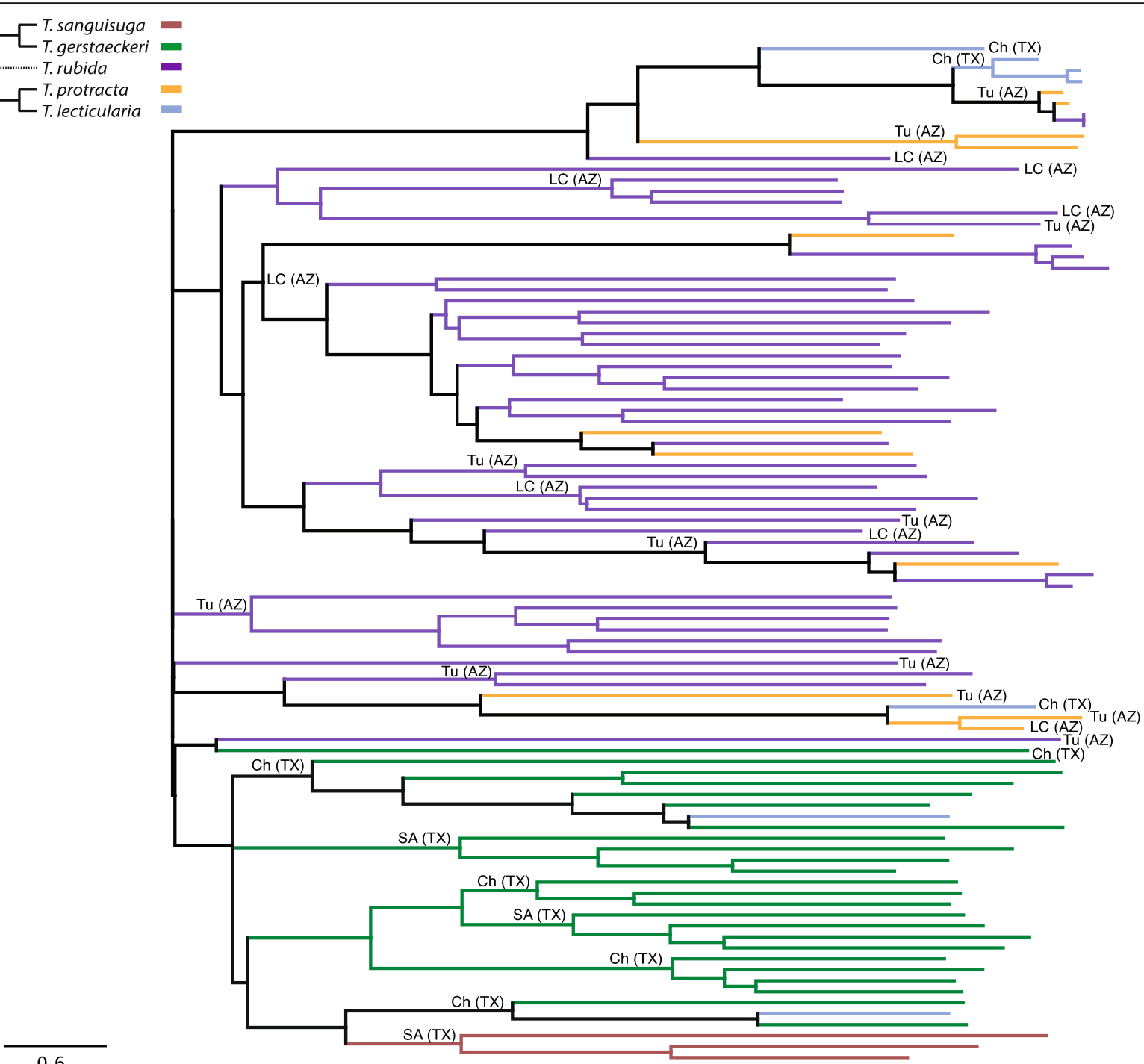

Fig. 5 The neighbour-joining tree of microbiome Bray-Curtis dissimilarities for 93 Triatoma early instar individuals, showing microbiome species specificity is determined by host phylogeny and geographic origin. The nodes are coloured according to host phylogeny. The simplified phylogenetic scheme for the five Triatoma species is based on Georgieva et al. [5] and Ibarra-Cerdeña et al. [82]. The dashed line reflects the uncertain position of T. rubida. Sample geographic origin is designated as follows: SA (TX) San Antonio, Texas; Ch (TX) Chaparral, Texas; Tu (AZ) University of Arizona Desert Station, Tucson, Arizona; LC (AZ) Las Cienegas National Conservation Area, Arizona

associated with all five individuals. These represented the genera Dietzia and Kitasatospora (Actinobacteria), Bacillus (Firmicutes), and Enterobacter (Proteobacteria).

Since microbiomes of hematophagous insects often contain acquired bacterial pathogens [83, 84], we examined our data for known pathogenic genera. Two OTUs assigned to the genus Bartonella were found in the "basic" and "decontam" datasets, one of which was retained in the "ultraclean" data. This Bartonella OTU was highly prevalent (51\%) among all $T$. protracta and T. rubida individuals. Using Bartonella gltA genespecific primers [85], we retrieved 272-bp sequences from T. rubida individuals sampled from LCNCA and UADS. We found $99-100 \%$ pairwise similarity between the sequences from UADS and Bartonella vinsonii isolates from $N$. albigula blood (available in GenBank; KJ719286-7). Bartonella gltA sequences retrieved from LCNCA samples were equally similar with Bartonella vinsonii isolates from unspecified rodents (AF148491, AF148493, AF148481), suggesting that T. rubida acquired pathogenic Bartonella from its' vertebrate hosts (Additional File 8). The representative sequences for $B$. vinsonii found here are available in GenBank under the following accession numbers MT112947-MT112949. 


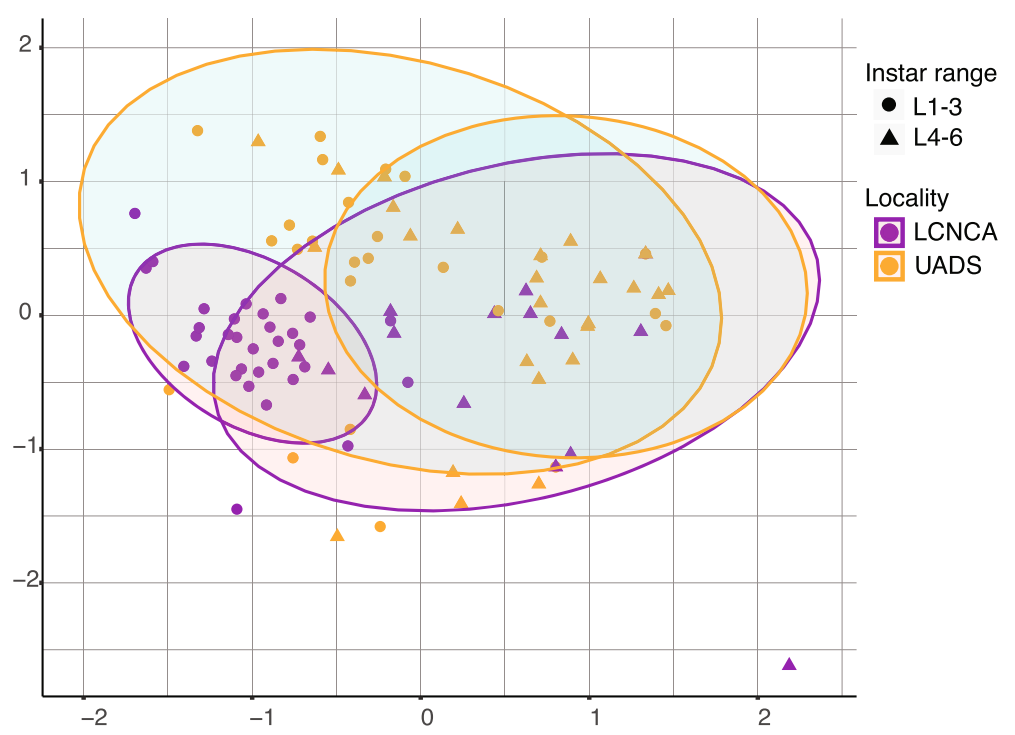

Fig. 6 NMDS visualisation of the microbiome differences in T. rubida from N. albigula nests, sampled in two different locations in southern Arizona (LCNCA and UADS; represented by different colours). The results are also grouped into early- and late-instar groups (represented by different shapes). The statistical ellipses were assigned using a 0.05 confidence interval

\section{Discussion}

A wide range of factors have been suggested to determine the microbiomes of triatomines. Species identity, ontogeny, sex, blood meal source, geographical origin, physiological state, and T. cruzi infection have all been implicated from the current literature [24, 43, 49, 50]. However, the actual importance of these factors remains inconclusive or controversial. To address this issue within a broader phylogenetic and geographic context allowing for more general conclusions, we designed the first large-scale investigation of triatomine microbiomes, sampling wild populations of five Triatoma species. Our data shows that microbiomes of wild triatomines are determined by three main factors: ontogeny, species specificity, and geographic origin, and are also influenced by pathogen uptake from their vertebrate hosts.

\section{Losing diversity: the main ontogenetic shift in microbiome composition}

Wild Triatoma microbiomes display dramatic compositional shifts from high diversity in first instars towards low diversity dominated by a single bacterium in adults. This general pattern, previously shown only from laboratory-reared Rhodnius prolixus [51], is well documented in T. rubida (our most sampled species) and indicated in three other species (T. gerstaeckeri, $T$. protracta, and T. lecticularia). Statistically, ontogeny is the most important explanatory factor of the microbiome dissimilarities found among T. rubida individuals. However, there are two caveats to this general pattern of microbiome diversity. Firstly, there is high amongindividual variation in richness reduction. For example, some L2s retained highly diverse microbiomes; others showed large reductions in richness reflected by single taxon dominance. The trend towards single taxon dominance increases in later developmental stages (L4 to L6). Among-individual variability was independent of engorgement status (scores are recorded in metadata), suggesting that it is not a product of host physiological state. Furthermore, it suggests that individual triatomines can maintain various different microbiome arrangements (a rich microbiome vs. one dominated by a single taxon). Secondly, Dietzia is clearly the dominant bacteria in most late-stage individuals of $T$. rubida, $T$. lecticularia, and T. protracta, but some of their microbiomes are dominated by other genera (Mycobacterium, Proteiniphilum). High single taxon prevalence in late ontogenetic stages likely reflects a real biological process rather than a methodological artefact (e.g., artificial overamplification). We base this assertion on three major points: (i) our positive control profiles did not indicate any major preferential amplification in the data; (ii) the non-random occurrence of this pattern, i.e., Dietzia dominates the late ontogenetic stages of three Triatoma species; and (iii) concordant results of Mann et al. [50] showing $65 \%$ of $T$. sanguisuga and $T$. gerstaeckeri adult microbiomes are dominated by a single bacterial taxon, often Bacillus or an unspecified Enterobacteriaceae.

Developmental stage has been recognised as a key determinant of microbiome composition in other arthropod vectors (e.g., ticks [86-88]). In Triatoma, we can only hypothesise about the mechanisms underlying the ontogenetic shift from taxon-rich microbiomes in early instars to single taxon-dominated microbiomes in adults. 
Ontogenetic reduction of microbiome diversity may be random or induced by a specific physiological state, e.g., the moulting process. Insects typically shed the foregut and hindgut linings during moulting, causing loss or suppression of symbiotic bacteria in the process [89-91]. The rich microbial community of Triatoma first instars (possibly acquired from their eggs $[92,93]$ ) may be periodically shed from the gut with each moulting. After five moulting events, adults thus possess a microbiome with significantly reduced richness. However, the relationship between ontogeny and microbiome composition has not been investigated in other hemimetabolous bloodfeeding insects (bed bugs or lice [21];), making generalisable conclusions between biologically similar taxa elusive. Similar examples of decreasing microbiome diversity can be found throughout the lifecycle of some holometabolous insects, e.g., dung beetles [94] and cabbage flies [95]. For holometabolous insects, ontogenetic changes in gut bacterial communities are generally explained by two main factors: the substantial remodelling of the gastrointestinal tract during metamorphosis [89] and different dietary needs between larvae and adults [94]. Although hemimetabolous Triatominae depend on a blood-based diet throughout their entire development, their preferences for accessory feeding strategies, especially haemolymphagy and coprophagy, may decrease over time and thus limit opportunities for any microbiome enrichment. Since gut microbiome analysis of natural populations requires killing the specimen, we cannot record microbiome shifts throughout ontogenetic development of a single individual, and thus cannot currently determine whether diversity loss is a permanent change to triatomine microbiomes.

\section{Origin of the microbiome bacteria: inheritance vs, environment}

The results shown in Figs. 2, 3, 4, 5, and 6 demonstrate that triatomine microbiome composition (especially in early instars) is shaped by host species and locality. For both variables, there was partial overlap in microbiome composition, likely due to the notable degree of amongindividual variability (discussed above). Species-specific differences were present even when multiple triatomine species were sampled in the same nest, showing that they are not caused by different environmental sources of bacteria. In theory, they could be explained by multiple different mechanisms, like specific maternally inherited bacteria or differential uptake and retention of environmental bacteria. Due to microbiome ontogenetic changes, most of the early stage diversity is lost in the later stages and these bacteria are therefore unlikely to be transmitted vertically. Thus, only the bacteria present in both early stages and adults remain possible candidates for maternal inheritance. To address this issue, we cross-referenced bacterial OTUs from late-stage (L6) and early-stage (L1/L2) triatomines captured from the same nest. One nest from Arizona contained early- and late-stage T. rubida, as did one nest in Texas for T. lecticularia. In both instances there were common genera within all individuals (13 in T. rubida, 4 in T. lecticularia). We hypothesise that these shared taxa are the most likely candidates for vertical transmission. Moreover, the majority of the candidate bacteria fall within Actinobacteria, shown to be maternally inherited in other true bugs and wasps [93, 96-98]. However, we cannot exclude other potential causes for the patterns found, such as host immune selective pressure favouring these bacterial taxa.

While the significance of maternal inheritance is unclear, the effect of environmental bacteria is more evident. A prominent component of environmental microbe acquisition is potential vertebrate pathogens in the blood meal. In some hematophagous arthropods, vertebrate pathogens have evolved into symbionts (e.g., Francisella in the Gulf Coast tick [84]). Others, like sheep keds (Melophagus ovinus [99]) and a single kissing bug species (Eratyrus mucronatus [100]), were found to carry Bartonella species of an unknown phenotype. In our data, Bartonella was the second most abundant taxa found in every life stage of T. rubida. Molecular analysis showed that the bacterium is a pathogen acquired from $N$. albigula. A possible phenomenon for future consideration is whether Bartonella is a transcriptionally active component of Triatoma microbiomes, or a transient taxon reacquired with each feeding. Mycobacterium, the sixth most abundant OTU within T. rubida, provides another potential example of a pathogen [101, 102] acquired from the environment. Strict vertical inheritance and environmental uptake (horizontal transmission) are biologically distinct modes of acquiring bacterial symbionts. Triatomines engage in accessory feeding behaviours that potentially interconnect these two sources, such as coprophagy and kleptohematophagy $[2,55]$. For instance, coprophagy is employed by first instar Rhodnius prolixus to acquire Rhodococcus rhodnii from parental faeces [103]. This form of symbiont acquisition is not strict maternal inheritance, because offspring do not acquire Rhodococcus in utero or from the mother's ovaries. Instead, coprophagous symbiont acquisition represents both "indirect" vertical transmission and environmental acquisition. Currently, we cannot determine whether Triatoma microbiome species specificity is due to transmission of maternally provided bacteria or genetically determined uptake of environmental bacteria (e.g., the lack of Dietzia in T. gerstaeckeri and $T$. sanguisuga could be linked to the host's close phylogenetic relationship), or a combination of both. 


\section{Dominant taxa and endosymbiosis}

The consensus for many arthropod vectors is strong reliance on obligate bacterial endosymbionts (e.g., Wigglesworthia in Glossina, Riesia in Pediculus lice, Coxiellalike symbiont in ticks [16-20]) that facilitate essential functions like vitamin synthesis and participate in blood meal breakdown. Triatomines appear to establish less intimate symbioses with extracellular bacteria in their gut lumen, instead of possessing obligate intracellular symbionts $[49,51,104]$. Some studies indicated that Rhodococcus (an extracellular symbiont) was important for successful development and reproduction of Rhodnius prolixus [105-107]. However, later molecular studies showed Rhodococcus is not omnipresent throughout Triatominae, and not even among other Rhodnius species [47, 108]. In our results, Dietzia, a bacterium closely related to Rhodococcus [109], is the dominant bacterium in late instars of T. rubida, T. protracta, and T. lecticularia. Dietzia has been described from other triatomine species [40, 41, 43, 46, 47, 51] and other hematophagous insects (Aedes albopictus [110], Glossina pallidipes [111]), suggesting it may be an important mutualist. However, unlike typical primary symbionts, Dietzia does not seem to be transmitted vertically. In contrast to its obvious dominance in later instars, the presence of Dietzia in L1 is questionable. The "decontam" dataset showed that five T. rubida L1s had 1-3 reads of Dietzia (from a median average of 1864 reads). Such low read numbers cannot be discriminated from marginal wellto-well contamination and do not provide evidence of Dietzia presence in first instars. Further studies with quantitative and in situ approaches are required to unequivocally determine the transmission mechanism and presence of Dietzia in first instar triatomines. One hypothesis is that individual bugs acquire Dietzia from other triatomines via "indirect" vertical transmission (analogous to the R. prolixus and Rhodococcus example described above [103]) or from the environment, strategies that have been found in other true bug (Heteroptera) species, including trophallaxis, egg smearing, and endosymbiont reacquisition from soil [93, 112-117]. To further investigate transmission and function of triatomine microbiomes, we will require tissue specific whole genome sequencing and functional transcriptomic studies.

\section{Consistency of the patterns: biology vs. methodology}

Previous studies on triatomine microbiomes have suggested various factors, including ontogeny $[48,51]$, species identity $[41,47,50,51,108]$, sex $[44,50]$, blood meal source [44], and T. cruzi infection [24, 43, 45, 49, 50], as determinants of microbiome composition, while another study claimed triatomine microbiomes have no determining factor [40]. Since many were based on limited sample size (e.g., $N=4$ in [40], $N=14$ in [44], $N$ $=20$ in [42], $N=9$ in [41], $N=29$ in [51]) and largely fragmented by host taxonomy, ontogeny, geographic origin, T. cruzi infection status, or were restricted to colony-reared bugs, it is difficult to draw comparative conclusions. We thus paid particular attention to our sampling design and molecular approach, ensuring that our study enabled multiple comparisons at different scales (i.e., different species from the same locality, different species from the same microhabitat, and different localities for the same species) across all ontogenetic stages. Furthermore, by introducing a novel method with a blocking primer (see the "Novel 18S rRNA gene blocking primer" section), we achieved greater sequencing depth of 16S rRNA amplicons in our mixed templates. As a result, this study presents multiple deterministic patterns consistent across several triatomine species for the first time.

Some of our findings contradict the patterns reported by other authors. The most conspicuous example is the ontogenetic decrease of microbiome diversity in North American species, which is supported by Mann et al. [50], but contrasts two studies on South American species [47, 48]. Oliveira et al. [48] reported an increase in microbiome diversity throughout ontogenetic development in T. sordida, and Waltmann et al. [47] found no ontogenetic effect in $T$. infestans. There are two possible reasons for these differences. One is biological, because the other studies worked with South American species and species-specific differences are a clear component of microbiome dissimilarity, as our results and the results of others show [41, 51, 108]. The other is methodological, since the design of Oliveira et al.'s [48] study involved pooled samples and therefore does not allow evaluation of individual microbiome composition in different ontogenetic stages. Considering the amongindividual compositional variability we observed, it is clear that pooling samples may have significantly distorted the profiles. A similar methodological artefact has been shown in mosquitoes [118]. The lack of ontogenetic differences in Waltmann et al. [47] could reflect the sample source (faeces) and incompleteness of the ontogenetic spectrum (L3 to adults only) rather than a real biological pattern in natural populations.

The importance of sampling the complete ontogenetic spectrum is well demonstrated by comparing our results with the recent survey of Mann et al. [50], which focused on T. sanguisuga and T. gerstaeckeri. By profiling microbiomes of 74 specimens, they also revealed a high degree of among-individual variability. However, since their study was based solely on adults, they reported weak species specificity, whereas we found that speciesspecific microbiome patterns were more pronounced in early instars. In addition, Mann et al. [50] found support 
for locality-based effects which corroborates our findings for T. rubida from multiple locations in southern Arizona. These examples show that there is some consistency across triatomine microbiome studies, at least regarding US species. However, the current paucity of data does not allow for broader cross-study comparisons. To reach more generalisable conclusions for all Triatominae, we require added breadth (more studies) and depth (metagenomics and transcriptomics) of molecular data.

\section{Conclusion}

This study has contributed key information on triatomine microbiomes, which constitutes a crucial component of their biology. We identified ontogenetic shift, species identity, and the environment as the major factors determining microbiome composition in natural populations of T. rubida, T. protracta, T. lecticularia, $T$. sanguisuga, and T. gerstaeckeri, thus observing consistent deterministic patterns across multiple triatomine species for the first time. We hypothesise that the high among-individual variability of Triatominae microbiome assemblages is produced by inconsistent uptake of environmental bacteria, including vertebrate pathogens, and multiple indirect bacterial transmission strategies. The epidemiological relevance of Triatominae and their microbiome communities both warrant more in-depth exploration for successful implementation of microbiome-based vector control strategies. To achieve this, we advocate that future studies are designed to allow comparison of detected patterns across different triatomine populations, species, biogeographic areas, and environments.

\section{Supplementary information}

Supplementary information accompanies this paper at https://doi.org/10. 1186/s40168-020-00921-x.

Additional File 1: Sample metadata including list of discarded contaminants.

Additional File 2: Methodological Supplement for $18 \mathrm{~S}$ rRNA gene blocking primer design and validation

Additional File 3: Results of ordination analyses based on 'basic' and 'decontam' datasets.

Additional File 4: Sample set phylogenetic background inferred from COXB sequences.

Additional File 5: Microbiome ontogenetic shift in other Triatoma sp.

Additional File 6: Significant difference in beta dispersion of the instar range groups (L1-L3 and L4-L6) calculated from the ultraclean dataset.

Additional File 7: NMDS analyses of $T$. rubida microbiomes from early instar (L1-L3) individuals found in different $N$. albigula nests at UADS.

Additional File 8: Bartonella phylogenetic analysis of gltA sequences retrieved from T. rubida.

\section{Acknowledgements}

We wish to thank Allison Hansen, Patrick Degnan, and Robert L. Smith for all their invaluable help and support in the USA. This research was performed with contributions from the Texas Park and Wildlife Management Department and the Bureau of Land Management (Gila District Office, Tucson, AZ).

\section{Authors' contributions}

EN designed the research. JJB, EN, SRR, and WR collected the field samples. JOS provided supplementary samples. EN and JZ designed the novel $18 \mathrm{~S}$ rRNA gene blocking primers. JZ, SRR, and EN performed the DNA isolation and library preparation. AP performed T. cruzi screening. GB performed Bartonella sequencing. JJB, EN, SRR, AP, and VH analysed the data and interpreted results. JJB, EN, SRR, and VH wrote the manuscript. All authors contributed to improving the manuscript. The authors read and approved the final manuscript.

\section{Funding}

This research was funded by the Czech Science Foundation, grant no. 31-18$24707 Y$ to E. Nováková. The funding body played no part in the study design, collection, analysis, an interpretation of data, or the writing of the manuscript.

\section{Availability of data and materials}

The datasets supporting the conclusions of this article and are included with the article and its additional files. A representative subsample of $c y t B$ sequences for all Triatoma species in this study were uploaded to NCBI (accession number MT239320-MT239329). Representative sequences for Bartonella vinsonii are available under the following accession numbers MT112947-MT112949. Raw, demultiplexed microbiome data for this study and 926X blocking primer validation were uploaded to ENA (project numbers: PRJEB36515 and PRJNA657483). Complete R code is available at https://github.com/apoosakkannu/MS-Triatominae.

Ethics approval and consent to participate Not applicable.

\section{Consent for publication}

Not applicable.

\section{Competing interests}

The authors declare that they have no competing interests.

\section{Author details}

${ }^{1}$ Faculty of Science, University of South Bohemia, Ceske Budejovice, Czech Republic. ${ }^{2}$ Institute of Entomology, Biology Centre of the Czech Academy of Sciences, Ceske Budejovice, Czech Republic. ${ }^{3}$ Southwestern Biological Institute, Tucson, AZ, USA. ${ }^{4}$ US Army Public Health Command-Central, JBSA Fort Sam, Houston, TX, USA. ${ }^{5}$ Institute of Parasitology, Biology Centre of the Czech Academy of Sciences, Ceske Budejovice, Czech Republic.

Received: 24 June 2020 Accepted: 9 September 2020

Published online: 11 October 2020

\section{References}

1. Bern C, Kjos S, Yabsley MJ, Montgomery SP. Trypanosoma cruzi and Chagas' disease in the United States. Clin Microbiol Rev. 2011;24:655-81.

2. Klotz SA, Dorn PL, Mosbacher M, Schmidt JO. Kissing bugs in the United States: risk for vector-borne disease in humans. Environ Health Insights. 2014:8(s2):EHI.S16003.

3. Curtis-Robles R, Auckland LD, Snowden KF, Hamer GL, Hamer SA. Analysis of over 1500 triatomine vectors from across the US, predominantly Texas, for Trypanosoma cruzi infection and discrete typing units. Infect Genet Evol. 2018;58:171-80.

4. Reisenman CE, Lawrence G, Guerenstein PG, Gregory T, Dotson E, Hildebrand JG. Infection of kissing bugs with Trypanosoma cruzi, Tucson, Arizona, USA. Emerg Infect Dis. 2010;16:400-5.

5. Georgieva AY, Gordon ERL, Weirauch C. Sylvatic host associations of Triatominae and implications for Chagas disease reservoirs: a review and new host records based on archival specimens. PeerJ. 2017;5:e3826.

6. Woody NC, Woody HB. American trypanosomiasis (Chagas' disease): first indigenous case in the United States. J Am Med Assoc. 1955;159:676-7.

7. Ochs DE, Hnilica VS, Moser DR, Smith JH, Kirchhoff LV. Postmortem diagnosis of autochthonous acute chagasic myocarditis by polymerase chain reaction amplification of a species-specific DNA sequence of Trypanosoma cruzi. Am J Trop Med Hyg. 1996;54:526-9.

8. Garcia MN, Burroughs H, Gorchakov R, Gunter SM, Dumonteil E, Murray KO, et al. Molecular identification and genotyping of Trypanosoma cruzi DNA in 
autochthonous Chagas disease patients from Texas, USA. Infect Genet Evol. 2017:49:151-6.

9. Gunter SM, Murray KO, Gorchakov R, Beddard R, Rossmann SN, Montgomery SP, et al. Likely autochthonous transmission of Trypanosoma cruzi to humans, south central Texas, USA. Emerg Infect Dis. 2017;23:500-3.

10. Beatty NL, Perez-Velez CM, Yaglom HD, Carson S, Liu E, Khalpey Zl, et al. Evidence of likely autochthonous transmission of Chagas disease in Arizona. Am J Trop Med Hyg. 2018;99:1534-6.

11. Brown JJ, Mihaljevic JR, Des Marteaux L, Hrček J. Metacommunity theory for transmission of heritable symbionts within insect communities. Ecol Evol. 2020;10:1703-21.

12. McLean AHC, Parker BJ, Hrček J, Henry LM, Godfray HCJ. Insect symbionts in food webs. Philos Trans R Soc B. 2016;371:20150325.

13. Oliver KM, Martinez AJ. How resident microbes modulate ecologicallyimportant traits of insects. Curr Opin Insect Sci. 2014:4:1-7.

14. Song SJ, Sanders JG, Baldassarre DT, Chaves JA, Johnson NS, Piaggio AJ, et al. Is there convergence of gut microbes in blood-feeding vertebrates? Philos Trans R Soc B Biol Sci. 2019;374:20180249.

15. Ribeiro JMC, Arcà B. Chapter 2 from sialomes to the sialoverse: an insight into salivary potion of blood-feeding insects. In: Advances in Insect Physiology. Academic Press; 2009. p. 59-118. doi:https://doi.org/10.1016/ S0065-2806(09)37002-2.

16. Akman L, Yamashita A, Watanabe H, Oshima K, Shiba T, Hattori M, et al. Genome sequence of the endocellular obligate symbiont of tsetse flies, Wigglesworthia glossinidia. Nat Genet. 2002;32:402-7.

17. Allen JM, Reed DL, Perotti MA, Braig HR. Evolutionary relationships of "Candidatus Riesia spp.," endosymbiotic Enterobacteriaceae living within hematophagous primate lice. Appl Environ Microbiol. 2007;73:1659-64.

18. Pais R, Lohs C, Wu Y, Wang J, Aksoy S. The obligate mutualist Wigglesworthia glossinidia influences reproduction, digestion, and immunity processes of its host, the tsetse fly. Appl Environ Microbiol. 2008; 74:5965-74.

19. Buysse M, Plantard O, McCoy KD, Duron O, Menard C. Tissue localization of Coxiella-like endosymbionts in three European tick species through fluorescence in situ hybridization. Ticks Tick-Borne Dis. 2019;10:798-804.

20. Smith TA, Driscoll T, Gillespie JJ, Raghavan R. A Coxiella-like endosymbiont Is a potential vitamin source for the lone star tick. Genome Biol Evol. 2015;7: 831-8.

21. Husnik F. Host-symbiont-pathogen interactions in blood-feeding parasites: nutrition, immune cross-talk and gene exchange. Parasitology. 2018;145: 1294-303.

22. Gaio A De O, Gusmão DS, Santos AV, Berbert-Molina MA, Pimenta PF, Lemos FJ. Contribution of midgut bacteria to blood digestion and egg production in Aedes aegypti (diptera: culicidae) (L.). Parasit Vectors. 2011;4:105.

23. Duron O, Gottlieb Y. Convergence of nutritional symbioses in obligate blood feeders. Trends Parasitol. 2020. https://doi.org/10.1016/j.pt.2020.07.007.

24. Castro DP, Moraes CS, Gonzalez MS, Ratcliffe NA, Azambuja P, Garcia ES. Trypanosoma cruzi immune response modulation decreases microbiota in Rhodnius prolixus gut and is crucial for parasite survival and development. PLoS One. 2012;7:e36591.

25. Azambuja P. Garcia ES, Ratcliffe NA. Gut microbiota and parasite transmission by insect vectors. Trends Parasitol. 2005;21:568-72.

26. Dennison NJ, Jupatanakul N, Dimopoulos G. The mosquito microbiota influences vector competence for human pathogens. Curr Opin Insect Sci. 2014;3:6-13.

27. Hegde S, Rasgon $\mathrm{JL}$, Hughes GL. The microbiome modulates arbovirus transmission in mosquitoes. Curr Opin Virol. 2015;15:97-102.

28. Geiger A, Fardeau M-L, Njiokou F, Ollivier B. Glossina spp. gut bacterial flora and their putative role in fly-hosted trypanosome development. Front Cell Infect Microbiol. 2013:3. doi:10.3389/fcimb.2013.00034.

29. Aksoy S, Weiss BL, Attardo GM. Trypanosome transmission dynamics in tsetse. Curr Opin Insect Sci. 2014:3:43-9.

30. Louradour I, Monteiro CC, Inbar E, Ghosh K, Merkhofer R, Lawyer P, et al. The midgut microbiota plays an essential role in sand fly vector competence for Leishmania major. Cell Microbiol. 2017;19:e12755.

31. Weiss BL, Wang J, Maltz MA, Wu Y, Aksoy S. Trypanosome infection establishment in the tsetse fly gut is influenced by microbiome-regulated host immune barriers. PLoS Pathog. 2013;9:e1003318.

32. Cirimotich CM, Ramirez JL, Dimopoulos G. Native microbiota shape insect vector competence for human pathogens. Cell Host Microbe. 2011;10:307-10
33. Saldaña MA, Hegde S, Hughes GL. Microbial control of arthropod-borne disease. Mem Inst Oswaldo Cruz. 2017;112:81-93.

34. Adair KL, Bost A, Bueno E, Kaunisto S, Kortet R, Peters-Schulze G, et al. Host determinants of among-species variation in microbiome composition in drosophilid flies. ISME J. 2020;14:217-29.

35. Lim SJ, Bordenstein SR. An introduction to phylosymbiosis. Proc R Soc B Biol Sci. 2020;287:20192900

36. Park R, Dzialo MC, Spaepen S, Nsabimana D, Gielens K, Devriese H, et al. Microbial communities of the house fly Musca domestica vary with geographical location and habitat. Microbiome. 2019;7:147.

37. Cohen H, McFrederick QS, Philpott SM. Environment shapes the microbiome of the blue orchard bee, Osmia lignaria. Microb Ecol. 2020. https://doi.org/10.1007/s00248-020-01549-y.

38. Wolz CRM, Yarwood SA, Grant EHC, Fleischer RC, Lips KR. Effects of host species and environment on the skin microbiome of Plethodontid salamanders. J Anim Ecol. 2018;87:341-53.

39. Ingala MR, Becker DJ, Holm JB, Kristiansen K, Simmons NB. Habitat fragmentation is associated with dietary shifts and microbiota variability in common vampire bats. Ecol Evol. 2019;9:6508-23.

40. Gumiel M, da Mota FF, Rizzo V de S, Sarquis O, de Castro DP, Lima MM, et al. Characterization of the microbiota in the guts of Triatoma brasiliensis and Triatoma pseudomaculata infected by Trypanosoma cruzi in natural conditions using culture independent methods. Parasit Vectors. 2015;8:245

41. Montoya-Porras LM, Omar T-C, Alzate JF, Moreno-Herrera CX, CadavidRestrepo GE. 16S rRNA gene amplicon sequencing reveals dominance of Actinobacteria in Rhodnius pallescens compared to Triatoma maculata midgut microbiota in natural populations of vector insects from Colombia. Acta Trop. 2018;178:327-32.

42. Lopez-Ordonez T, Flores-López CA, Montejo-Lopez R, Cruz-Hernandez A, Conners EE. Cultivable bacterial diversity in the gut of the Chagas disease vector Triatoma dimidiata: identification of possible bacterial candidates for a paratransgenesis approach. Front Ecol Evol. 2018;5.

43. Díaz S, Villavicencio B, Correia N, Costa J, Haag KL. Triatomine bugs, their microbiota and Trypanosoma cruzi: asymmetric responses of bacteria to an infected blood meal. Parasit Vectors. 2016:9:636

44. Dumonteil E, Ramirez-Sierra M-J, Pérez-Carrillo S, Teh-Poot C, Herrera C, Gourbière $S$, et al. Detailed ecological associations of triatomines revealed by metabarcoding and next-generation sequencing: implications for triatomine behavior and Trypanosoma cruzi transmission cycles. Sci Rep. 2018;8:4140.

45. Dumonteil E, Pronovost H, Bierman EF, Sanford A, Majeau A, Moore R, et al. Interactions among Triatoma sanguisuga blood feeding sources, gut microbiota and Trypanosoma cruzi diversity in southern Louisiana. Mol Ecol. 2020. https://doi.org/10.1111/mec.15582.

46. Carels N, Gumiel M, da Mota FF, de Carvalho Moreira CJ, Azambuja P. A metagenomic analysis of bacterial microbiota in the digestive tract of triatomines. Bioinforma Biol Insights. 2017;11:1177932217733422.

47. Waltmann A, Willcox AC, Balasubramanian S, Mayori KB, Guerrero SM, Sanchez RSS, et al. Hindgut microbiota in laboratory-reared and wild Triatoma infestans. PLoS Negl Trop Dis. 2019;13:e0007383.

48. Oliveira JL, Cury JC, Gurgel-Gonçalves R, Bahia AC, Monteiro FA. Fieldcollected Triatoma sordida from central Brazil display high microbiota diversity that varies with regard to developmental stage and intestinal segmentation. PLoS Negl Trop Dis. 2018;12:e0006709.

49. Orantes LC, Monroy C, Dorn PL, Stevens L, Rizzo DM, Morrissey L, et al. Uncovering vector, parasite, blood meal and microbiome patterns from mixed-DNA specimens of the Chagas disease vector Triatoma dimidiata. PLoS Negl Trop Dis. 2018;12:e0006730.

50. Mann AE, Mitchell EA, Zhang Y, Curtis-Robles R, Thapa S, Hamer SA, et al. Comparison of the bacterial gut microbiome of North American Triatoma spp. with and without Trypanosoma cruzi. Front Microbiol. 2020;11. doi:10. 3389/fmicb.2020.00364.

51. Rodríguez-Ruano SM, Škochová V, Rego ROM, Schmidt JO, Roachell W, Hypša V, et al. Microbiomes of North American Triatominae: the grounds for Chagas disease epidemiology. Front Microbiol. 2018;9:1167.

52. Kieran TJ, Arnold KMH, Thomas JC, Varian CP, Saldaña A, Calzada JE, et al. Regional biogeography of microbiota composition in the Chagas disease vector Rhodnius pallescens. Parasit Vectors. 2019;12:504.

53. Alves $\mathrm{CL}$, Araujo RN, Gontijo NF, Pereira MH. Importance and physiological effects of hemolymphagy in triatomines (Hemiptera: Reduviidae). J Med Entomol. 2011;48:372-81. 
54. Durán $P$, Siñani $E$, Depickère $S$, Durán $P$, Siñani $E$, Depickère $S$. On triatomines, cockroaches and haemolymphagy under laboratory conditions: new discoveries. Mem Inst Oswaldo Cruz. 2016;111:605-13.

55. Sandoval CM, Joya MI, Gutierez R, Angulo VM. Cleptohaematophagy of the triatomine bug Belminus herreri. Med Vet Entomol. 2000;14:100-1.

56. Schmidt JO, Dorn PL, Klotz SA. Second-best is better than nothing: cockroaches as a viable food source for the kissing bug Triatoma recurva (Hemiptera: Reduviidae). J Med Entomol. 2019;56:651-5.

57. Colman DR, Toolson EC, Takacs-Vesbach CD. Do diet and taxonomy influence insect gut bacterial communities? Mol Ecol. 2012;21:5124-37.

58. Yun J-H, Roh SW, Whon TW, Jung M-J, Kim M-S, Park D-S, et al. Insect gut bacterial diversity determined by environmental habitat, diet, developmental stage, and phylogeny of host. Appl Environ Microbiol. 2014; 80:5254-64.

59. Nováková E, Woodhams DC, Rodríguez-Ruano SM, Brucker RM, Leff JW, Maharaj A, et al. Mosquito microbiome dynamics, a background for prevalence and seasonality of West Nile virus. Front Microbiol. 2017:8:526.

60. Monteiro FA, Barrett TV, Fitzpatrick S, Cordon-Rosales C, Feliciangeli D, Beard CB. Molecular phylogeography of the Amazonian Chagas disease vectors Rhodnius prolixus and R. robustus. Mol Ecol. 2003;12:997-1006.

61. Lyman DF, Monteiro FA, Escalante AA, Cordon-Rosales C, Wesson DM, Dujardin JP, et al. Mitochondrial DNA sequence variation among triatomine vectors of Chagas' disease. Am J Trop Med Hyg. 1999;60:377-86.

62. Pfeiler E, Bitler BG, Ramsey JM, Palacios-Cardiel C, Markow TA. Genetic variation, population structure, and phylogenetic relationships of Triatoma rubida and T. recurva (Hemiptera: Reduviidae: Triatominae) from the Sonoran Desert, insect vectors of the Chagas' disease parasite Trypanosoma cruzi. Mol Phylogenet Evol. 2006;41:209-21.

63. Darriba D, Taboada GL, Doallo R, Posada D. jModelTest 2: more models, new heuristics and parallel computing. Nat Methods 2012:9:772-772.

64. Moser DR, Kirchhoff LV, Donelson JE. Detection of Trypanosoma cruzi by DNA amplification using the polymerase chain reaction. J Clin Microbiol. 1989;27:1477-82.

65. Lima L, Espinosa-Álvarez O, Ortiz PA, Trejo-Varón JA, Carranza JC, Pinto CM, et al. Genetic diversity of Trypanosoma cruzi in bats, and multilocus phylogenetic and phylogeographical analyses supporting Tcbat as an independent DTU (discrete typing unit). Acta Trop. 2015; 151:166-77.

66. Parada AE, Needham DM, Fuhrman JA. Every base matters: assessing small subunit rRNA primers for marine microbiomes with mock communities, time series and global field samples. Environ Microbiol. 2016;18:1403-14.

67. Quince C, Lanzen A, Davenport RJ, Turnbaugh PJ. Removing noise from pyrosequenced amplicons. BMC Bioinformatics. 2011;12:38.

68. Vestheim $\mathrm{H}$, Jarman SN. Blocking primers to enhance PCR amplification of rare sequences in mixed samples - a case study on prey DNA in Antarctic krill stomachs. Front Zool. 2008:5:12.

69. Wilcox TM, McKelvey KS, Young MK, Jane SF, Lowe WH, Whiteley AR, et al. Robust detection of rare species using environmental DNA: the importance of primer specificity. PLoS One. 2013;8. https://doi.org/10.1371/journal.pone. 0059520.

70. Edgar RC. UPARSE: highly accurate OTU sequences from microbial amplicon reads. Nat Methods. 2013;10:996-8.

71. Camacho C, Coulouris G, Avagyan V, Ma N, Papadopoulos J, Bealer K, et al. BLAST+: architecture and applications. BMC Bioinformatics. 2009:10:421.

72. Quast C, Pruesse E, Yilmaz P, Gerken J, Schweer T, Yarza P, et al. The SILVA ribosomal RNA gene database project: improved data processing and webbased tools. Nucleic Acids Res. 2013;41:D590-6.

73. Caporaso JG, Kuczynski J, Stombaugh J, Bittinger K, Bushman FD, Costello EK, et al. QIIME allows analysis of high-throughput community sequencing data. Nat Methods. 2010;7:335-6.

74. Bokulich NA, Subramanian S, Faith JJ, Gevers D, Gordon Jl, Knight R, et al. Quality-filtering vastly improves diversity estimates from Illumina amplicon sequencing. Nat Methods. 2013;10:57-9.

75. Davis NM, Proctor DM, Holmes SP, Relman DA, Callahan BJ. Simple statistical identification and removal of contaminant sequences in marker-gene and metagenomics data. Microbiome. 2018;6:226.

76. Oksanen J, Blanchet FG, Friendly M, Kindt R, Legendre P, McGlinn D, et al. vegan: Community Ecology Package. 2019. https://CRAN.R-project.org/ package =vegan
77. McMurdie PJ, Holmes S. phyloseq: An R package for reproducible interactive analysis and graphics of microbiome census data. PLoS ONE. 2013;8:e61217.

78. R Core Team. R: A language and environment for statistical computing. Vienna, Austria; 2019. https://www.R-project.org/.

79. Goslee SC, Urban DL. The ecodist package for dissimilarity-based analysis of ecological data. J Stat Softw. 2007;022. https://ideas.repec.org/a/jss/jstsof/ v022i07.html. Accessed 2 Jan 2020.

80. QGIS Development Team. QGIS geographic information system. Open Source Geospatial Foundation; 2019. http://qgis.org.

81. Legendre $P$, Cáceres MD. Beta diversity as the variance of community data: dissimilarity coefficients and partitioning. Ecol Lett. 2013;16:951-63.

82. Ibarra-Cerdeña CN, Zaldívar-Riverón A, Peterson AT, Sánchez-Cordero V, Ramsey JM. Phylogeny and niche conservatism in North and Central American triatomine bugs (Hemiptera: Reduviidae: Triatominae), vectors of Chagas' disease. PLoS Negl Trop Dis. 2014;8. https://doi.org/10.1371/journal. pntd.0003266

83. Cohen C, Toh E, Munro D, Dong Q, Hawlena H. Similarities and seasonal variations in bacterial communities from the blood of rodents and from their flea vectors. ISME J. 2015;9:1662-76.

84. Gerhart JG, Moses AS, Raghavan R. A Francisella-like endosymbiont in the Gulf Coast tick evolved from a mammalian pathogen. Sci Rep. 2016;6:1-6.

85. Norman AF, Regnery R, Jameson P, Greene C, Krause DC. Differentiation of Bartonella-like isolates at the species level by PCR-restriction fragment length polymorphism in the citrate synthase gene. J Clin Microbiol. 1995;33: 1797-803.

86. Moreno CX, Moy F, Daniels TJ, Godfrey HP, Cabello FC. Molecular analysis of microbial communities identified in different developmental stages of Ixodes scapularis ticks from Westchester and Dutchess Counties, New York. Environ Microbiol. 2006:8:761-72.

87. Noda H, Munderloh UG, Kurtti TJ. Endosymbionts of ticks and their relationship to Wolbachia spp. and tick-borne pathogens of humans and animals. Appl Environ Microbiol. 1997;63:3926-32.

88. Zolnik CP, Prill RJ, Falco RC, Daniels TJ, Kolokotronis S-O. Microbiome changes through ontogeny of a tick pathogen vector. Mol Ecol. 2016;25: 4963-77.

89. Hammer TJ, Moran NA. Links between metamorphosis and symbiosis in holometabolous insects. Philos Trans R Soc B Biol Sci. 2019;374: 20190068.

90. Kim JK, Han SH, Kim C-H, Jo YH, Futahashi R, Kikuchi Y, et al. Moltingassociated suppression of symbiont population and up-regulation of antimicrobial activity in the midgut symbiotic organ of the RiptortusBurkholderia symbiosis. Dev Comp Immunol. 2014;43:10-4.

91. Nalepa CA. What kills the hindgut flagellates of lower termites during the host molting cycle? Microorganisms. 2017;5:82.

92. Meng L, Li X, Cheng $X$, Zhang H. 16S rRNA gene sequencing reveals a shift in the microbiota of Diaphorina citri during the psyllid life cycle. Front Microbiol. 2019;10. https://doi.org/10.3389/fmicb.2019.01948.

93. Kaltenpoth $\mathrm{M}$, Winter SA, Kleinhammer A. Localization and transmission route of Coriobacterium glomerans, the endosymbiont of pyrrhocorid bugs. FEMS Microbiol Ecol. 2009;69:373-83.

94. Suárez-Moo P, Cruz-Rosales M, Ibarra-Laclette E, Desgarennes D, Huerta C, Lamelas A. Diversity and composition of the gut microbiota in the developmental stages of the dung beetle Copris incertus Say (Coleoptera, Scarabaeidae). Front Microbiol. 2020;11. https://doi.org/10.3389/fmicb.2020. 01698.

95. van den Bosch TJM, Welte CU. The microbial diversity of cabbage pest Delia radicum across multiple life stages. Front Microbiol. 2020;11. https://doi.org/ 10.3389/fmicb.2020.00315.

96. Kaltenpoth M, Göttler W, Herzner G, Strohm E. Symbiotic bacteria protect wasp larvae from fungal infestation. Curr Biol. 2005;15:475-9.

97. Kaltenpoth M, Roeser-Mueller K, Koehler S, Peterson A, Nechitaylo TY, Stubblefield JW, et al. Partner choice and fidelity stabilize coevolution in a Cretaceous-age defensive symbiosis. Proc Natl Acad Sci. 2014;111: 6359-64.

98. Kaltenpoth M, Engl T. Defensive microbial symbionts in Hymenoptera. Funct Ecol. 2014;28:315-27.

99. Nováková E, Husník F, Šochová E, Hypša V. Arsenophonus and Sodalis symbionts in louse flies: an analogy to the Wigglesworthia and Sodalis system in tsetse flies. Appl Environ Microbiol. 2015;81:6189-99. 
100. Laroche M, Berenger J-M, Mediannikov O, Raoult D, Parola P. Detection of a potential new Bartonella species "Candidatus Bartonella rondoniensis" in human biting kissing bugs (Reduviidae; Triatominae). PLoS Negl Trop Dis. 2017;11:e0005297.

101. Nessar R, Cambau E, Reyrat JM, Murray A, Gicquel B. Mycobacterium abscessus: a new antibiotic nightmare. J Antimicrob Chemother. 2012;67: 810-8.

102. Getahun H, Matteelli A, Chaisson RE, Raviglione M. Latent Mycobacterium tuberculosis infection. N Engl J Med. 2015;372:2127-35.

103. Eichler S, Schaub GA. Development of symbionts in triatomine bugs and the effects of infections with trypanosomatids. Exp Parasitol. 2002;100:17-27.

104. Beard CB, Cordon-Rosales C, Durvasula RV. Bacterial symbionts of the Triatominae and their potential use in control of Chagas disease transmission. Annu Rev Entomol. 2002;47:123-41.

105. Wigglesworth VB. Symbiotic bacteria in a blood-sucking insect, Rhodnius prolixus Stål. (Hemiptera, Triatomidae). Parasitology. 1936;28:284-9.

106. Harington JS. Synthesis of thiamine and folic acid by Nocardia rhodnii, the micro-symbiont of Rhodnius prolixus. Nature. 1960;188:1027-8.

107. Hill P, Campbell JA, Petrie IA, Pollock MR. Rhodnius prolixus and its symbiotic actinomycete: a microbiological, physiological and behavioural study. Proc R Soc Lond B Biol Sci. 1976;194:501-25.

108. da Mota FF, Marinho LP, Moreira CJ De C, Lima MM, Mello CB, Garcia ES, et al. Cultivation-independent methods reveal differences among bacterial gut microbiota in triatomine vectors of Chagas disease. PLoS Negl Trop Dis. 2012;6. https://doi.org/10.1371/journal.pntd.0001631.

109. Koerner RJ, Goodfellow M, Jones AL. The genus Dietzia: a new home for some known and emerging opportunist pathogens. FEMS Immunol Med Microbiol. 2009;55:296-305.

110. Yadav KK, Chandel K, Bora A, Veer V. Isolation and characterization of Dietzia maris from midgut of Aedes albopictus: a suitable candidate for paratransgenesis. Int J Mosq Res. 2015;2:7-12.

111. Malele I, Nyingilili H, Lyaruu E, Tauzin M, Bernard Ollivier B, Cayol J-L, et al. Bacterial diversity obtained by culturable approaches in the gut of Glossina pallidipes population from a non sleeping sickness focus in Tanzania: preliminary results. BMC Microbiol. 2018;18:164.

112. Kaiwa N, Hosokawa T, Nikoh N, Tanahashi M, Moriyama M, Meng X-Y, et al. Symbiont-supplemented maternal investment underpinning host's ecological adaptation. Curr Biol. 2014;24:2465-70.

113. Kikuchi Y, Hosokawa T, Fukatsu T. Insect-microbe mutualism without vertical transmission: a stinkbug acquires a beneficial gut symbiont from the environment every generation. Appl Environ Microbiol. 2007;73:4308-16

114. Hosokawa T, Ishii Y, Nikoh N, Fujie M, Satoh N, Fukatsu T. Obligate bacterial mutualists evolving from environmental bacteria in natural insect populations. Nat Microbiol. 2016;1:15011.

115. Nikoh N, Hosokawa T, Moriyama M, Oshima K, Hattori M, Fukatsu T. Evolutionary origin of insect-Wolbachia nutritional mutualism. Proc Natl Acad Sci. 2014;111:10257-62.

116. Engel P, Moran NA. The gut microbiota of insects - diversity in structure and function. FEMS Microbiol Rev. 2013;37:699-735.

117. Bistolas KSI, Sakamoto RI, Fernandes JAM, Goffredi SK. Symbiont polyphyly, co-evolution, and necessity in pentatomid stinkbugs from Costa Rica. Front Microbiol. 2014;5. https://doi.org/10.3389/fmicb.2014.00349.

118. Rodríguez-Ruano SM, Juhanáková E, Vávra J, Nováková E. Methodological insight into mosquito microbiome studies. Front Cell Infect Microbiol. 2020; 10. https://doi.org/10.3389/fcimb.2020.00086.

\section{Publisher's Note}

Springer Nature remains neutral with regard to jurisdictional claims in published maps and institutional affiliations.

Ready to submit your research? Choose BMC and benefit from:

- fast, convenient online submission

- thorough peer review by experienced researchers in your field

- rapid publication on acceptance

- support for research data, including large and complex data types

- gold Open Access which fosters wider collaboration and increased citations

- maximum visibility for your research: over $100 \mathrm{M}$ website views per year

At BMC, research is always in progress.

Learn more biomedcentral.com/submissions 